\title{
Mitotic kinesin-like protein 1, regulated by FOXM1, promotes triple negative breast cancer progression via activating Wnt/ $\beta$-catenin pathway
}

Zhi Li

Nanjing Medical University

Hai-Yan Yang

Nanjing Medical University

Xiao-Lan Zhang

Nanjing Medical University

Xu Zhang

Nanjing Medical University

Yu-Zhou Huang

Nanjing Medical University

Xin-Yuan Dai

Nanjing Medical University

Liang Shi

Nanjing Medical University

Guo-Ren Zhou

Nanjing Medical University

Ji-Fu Wei

Nanjing Medical University

Qiang Ding ( $\nabla$ dingqiang@njmu.edu.cn )

Jiangsu Province Hospital and Nanjing Medical University First Affiliated Hospital

https://orcid.org/0000-0003-1499-6200

\section{Research Article}

Keywords: MKLP1, Wnt/ $\beta$-catenin pathway, FOXM1, WDR5, H3K4me3, triple negative breast cancer

Posted Date: January 13th, 2022

DOI: https://doi.org/10.21203/rs.3.rs-1228723/v1

License: (c) (1) This work is licensed under a Creative Commons Attribution 4.0 International License.

Read Full License 


\section{Abstract}

Background: Triple negative breast cancer (TNBC) is highly malignant and has a worse prognosis, compared with other subtypes of breast cancer due to the absence of therapeutic targets. MKLP1 plays a crucial role in tumorigenesis and cancer progression. However, the role of MKLP1 in triple negative breast cancer and the underlying mechanism remain unknown. The study aimed to elucidate the biological function regulatory mechanism of MKLP1 in triple negative breast cancer

Methods: Quantitative real-time PCR and Western blotting were used to determine the MKLP1 expression in breast cancer tissues and cell lines. Then, functional experiments in vitro and in vivo were performed to investigate the effects of MKLP1 on tumor growth and metastasis in triple negative breast cancer. Chromatin immunoprecipitation assay was conducted to illustrate the potential regulatory mechanisms of MKLP1 in triple negative breast cancer.

Results: We found that MKLP1 was significantly up-regulated and associated with poor prognosis in triple negative breast cancer. MKLP1 could promote triple negative breast cancer proliferation, migration and invasion in vitro and in vivo. MKLP1 could activate Wnt/ $\beta$-catenin pathway and promote EMT progression. In addition, FOXM1, upregulated by WDR5 via H3K4me3 modification, directly bound to the promoter of MKLP1 gene to promote its transcription and accelerated TNBC progression via Wnt/ $\beta$ catenin pathway. Both of small inhibitor of FOXM1 and WDR5 could inhibit TNBC progression.

Conclusions: Our findings elucidate WDR5/FOXM1/MKLP1/Wnt/ $\beta$-catenin axis is associated with TNBC progression and may provide a novel and promising therapeutic target for TNBC treatment.

\section{Background:}

Breast cancer is the most common malignant tumor in women and the leading cause of death among women [1, 2]. It is mainly divided into four subtypes: Luminal A, Luminal B, epidermal growth factor receptor (HER) $2^{+}$and triple negative breast cancer (TNBC) by the biomarkers estrogen receptor (ER), progesterone receptor (PR) and HER2. The TNBC makes up 10-20\% of all breast cancers, lacking all of the biomarkers [3]. The prognosis of TNBC is significantly worse than other subtypes of breast cancer due to the absence of therapeutic targets [4]. It is urgent to discover new molecular targets to improve TNBC patients' prognosis.

MKLP1, which belongs to the kinesin superfamily consists of 45 family proteins [5], is a component of the central spindlin complex and involved in the cytokinesis [6-8]. It plays crucial roles in multiple normal cellular biological activities, such as cytoplasm separation in mitosis and cytokinesis [8, 9]. Moreover, previous studies demonstrated MKLP1 participated in the occurrence of multiple cancers, including gastric cancer, bladder cancer, lung cancer and pancreatic ductal adenocarcinoma [10-14]. In gastric cancer, MKLP1 promotes cancer cells proliferation via direct interaction with Amer1 [10]. In pancreatic ductal adenocarcinoma, overexpression of MKLP1 predicts poor prognosis [13]. However, the functional role of MKLP1 and the underlying mechanism in TNBC remain unknown. 
Epithelial-mesenchymal transition (EMT) is associated with the tumorigenic process of many cancers [15]. Several studies have illustrated EMT is involved in TNBC progression [16, 17]. Moreover, accumulating evidence have demonstrated signaling pathways play crucial roles in many cancers, such as PI3K/AKT [18], JAK/STAT [19], Wnt/ $\beta$-catenin [20], Hedgehog [21] signaling pathways and so on. Previous studies have illustrated that dysregulation of Wnt/ $\beta$-catenin pathway is involved in different types of cancers [22-25]. Additionally, it is associated with TNBC progression [26, 27], including proliferation, metastasis, and may be a potential therapeutic target for TNBC treatment [28]. However, the molecular basis for the dysregulation remains unclear.

In our study, we showed MKLP1 was obviously upregulated in TNBC and downregulated MKLP1 suppressed proliferation and metastasis of TNBC cells. In addition, downregulated MKLP1 inhibited EMT progression and Wnt/ $\beta$-catenin pathway. Moreover, our results demonstrated FOXM1, upregulated by WDR5 via H3K4me3 modification, upregulated MKLP1 expression via promoting MKLP1 transcription. Our findings elucidate WDR5/FOXM1/MKLP1/Wnt/ $\beta$-catenin axis is associated with TNBC progression and may provide a novel and promising therapeutic target.

\section{Methods:}

\section{Clinical tissue samples}

Human TNBC tissues and paired adjacent normal tissues were collected from the First Affiliated Hospital of Nanjing Medical University. All patients received no neoadjuvant therapy. All tissues were frozen in liquid nitrogen immediately after resection and stored at $-80^{\circ} \mathrm{C}$. The patients all provided written informed consent and the study was approved by the ethical committee of the First Affiliated Hospital of Nanjing Medical University.

\section{Bioinformatic analysis}

The different expression genes in TNBC were analyzed by GEO2R with the datasets deposited in GEO. The expression of MKLP1 in different subtypes of breast cancer was analyzed by UALCAN [29]. Relapsefree survival of TNBC was analyzed by Kaplan-Meier Plotter (https://kmplot.com/analysis/). CancerSEA and CGPE were used to analyze the biological function of MKLP1 [30, 31]. Correlation between genes was analyzed by TIMER [32]. Cistrome was used to predict the transcription factor and histone modification [33]. CHIP-Seq data analysis and visualization were conducted by TFmapper [34].

\section{Cell lines and cell culture}

All breast cancer cell lines including MCF-7, ZR-75-1, BT474, MDA-MB-231, MDA-MB-468, HCC1806 and normal human breast epithelial cell (MCF10A) were obtained from the American Tissue Culture Collection (ATCC, USA). SUM1315 cell line was provided by Stephen Ethier (University of Michigan). HCC1806 was cultured in RPMI-1640 medium (Gibco, USA), other cell lines were cultured in complete high glucose Dulbecco's modified Eagle medium (DMEM) (Gibco, USA), containing 10\% fetal bovine serum, $100 \mu \mathrm{g} / \mathrm{ml}$ penicillin-streptomycin (Hyclone, USA) at $37^{\circ} \mathrm{C}$ with $5 \% \mathrm{CO}_{2}$. 


\section{RNA isolation and quantitative real time-polymerase chain reaction (qRT-PCR)}

Total RNA was isolated using Trizol reagent (TaKaRa, Japan), and 1000 ng RNA was reverse-transcribed to cDNA using HiScript Q RT SuperMix (Vazyme, China). The qRT-PCR was performed using AceQ qPCR SYBR Green Master Mix (Vazyme, China) and GAPDH was used as endogenous control. The specific primers used are listed in Table S1

\section{Western blotting}

Proteins were extracted and electrophoresed on a $10 \%$ sodium dodecyl sulfate polyacrylamide (SDSPAGE) gels and transferred onto a polyvinylidene fluoride (PVDF) membrane (Millipore, USA). Then membranes were blocked in QuickBlock ${ }^{\text {TM }}$ blocking buffer (Beyotime, China) for $20 \mathrm{~min}$ and incubated with primary antibodies overnight at $4^{\circ} \mathrm{C}$. The membranes were then incubated for $2 \mathrm{~h}$ in appropriate secondary antibodies after membranes were washed 10 min three times with Tris buffered saline Tween (TBST). The ECL chemiluminescent reagent (Millipore, USA) was used to visualize proteins

\section{Lentivirus transfection and small interfering}

Cells were transfected with lentivirus (Genepharm, China) to downregulate MKLP1 expression. Puromycin $(3 \mu \mathrm{g} / \mathrm{ml})$ was used to select the stable cells for two weeks. The specific small interfering RNAs (siRNAs) for FOXM1 and WDR5 were purchased from GenePharma. The human MKLP1 and FOXM1 overexpression PCMV3-MKLP1 and pCMV3-FOXM1 vectors were obtained from Obio Technology. The sequences of siRNAs and RNA oligonucleotides were listed in Table S2.

\section{Cell proliferation assay}

The Cell Counting Kit-8 (CCK-8; Vazyme, China) was used to detect cell proliferation according to the manufacturer's protocol, and cell colony formation ability was assessed as described previously [35].

\section{5-ethynyl-2'-deoxyuridine (EdU) assay}

The cells were cultured in 96 -well plates $\left(2 \times 10^{4}\right.$ cells/well) for $24 \mathrm{~h}$, incubated with EdU at $37^{\circ} \mathrm{C}$ for $2 \mathrm{~h}$, and then nuclei were stained with 4',6-diamid-ino-2- phenylindole (DAPI), and the cells were visualized under a fluorescence microscope (Nikon, Japan).

\section{Wound healing assay and transwell assay}

Cell migration and invasion abilities were measured by the wound healing assay and transwell assay as reported previously [36].

\section{Chromatin immunoprecipitation assay (ChIP)}

ChIP assays were performed using chromatin immunoprecipitation kits (17-371, EZ-ChIP, Millipore) according to the manufacturer's instructions as in previous reports [37].

\section{Chemical reagents}


FOXM1 inhibitor (Thiostrepton) and WDR5 inhibitor (OICR-9429) were purchased from MedChemexpress (Monmouth Junction, NJ, USA). The regents were dissolved in dimethyl sulfoxide (DMSO) to generate the stock solution. For functional assays, the stock solution was diluted with culture medium. The working concentrations of thiostrepton and OICR-9429 were $5 \mu \mathrm{M}$ and $10 \mu \mathrm{M}$ respectively.

\section{Animal study}

All animal experiments were conducted according to the guidelines of Institutional Animal Care and Use Committee of the Nanjing Medical University. Twenty-four female BALB/c nude mice (aged 4 weeks, 1822g) were randomly divided into 4 groups (MDA-MB-231-sh-NC, MDA-MB-231-sh-MKLP1, SUM1315-shNC, SUM1315-sh-MKLP1). Stable cells or control cells ( $1 \times 10^{6}$ cells in $0.1 \mathrm{~mL}$ PBS) was subcutaneously injected into mammary fat pads of the mice and the growth of tumors was followed up every week. Tumor volume was measured every week using a caliper, calculated as (length $\times$ width2)/2. After 4 weeks, mice were sacrificed and checked for final tumor weight. For metastasis assay, stable cells were injected into the tail vein of each mouse. After 8 weeks, mice were sacrificed and examined for lung metastasis.

\section{Statistical analysis}

The statistical analysis was performed by SPSS software (Version 24.0) and GraphPad Prism (Version 8.0), and presented as mean \pm standard deviation (SD). All data were analyzed by two-tailed Student's ttest or ANOVA. Each experiment was repeated at least three times, and $p<0.05$ was considered statistically significant.

\section{Results:}

\section{MKLP1 is upregulated in TNBC tissues and cell lines}

We first explored the different expression genes in TNBC using the published data deposited in GEO. MKLP1 was significantly up-regulated in TNBC (Fig. 1A and S1A-E). We then analyzed a public microarray dataset (GSE41313) including gene expression data of 52 kinds of breast cancer cell lines. The result showed MKLP1 was significantly upregulated in TNBC cell lines compared with luminal cell lines (Fig. 1B). The findings were consolidated by the analysis of two other microarray datasets (GSE5460, GSE1456) and TCGA data (Fig. 1C-E and S1F). Moreover, patients with low MKLP1 expression showed better prognosis (Fig. 1F). MKLP1 expression was also determined in different subtypes of breast cancer cell lines and normal human breast cell lines by qRT-PCR and western blot analysis. However, MKLP1 mRNA expression was only highly expressed in TNBC cell lines, compared to the human normal breast cell line. While protein levels were highly expressed in all tumor cell lines (Fig. 1G). We next detected MKLP1 expression in 38 paired tumor tissues and in corresponding adjacent tissues from TNBC patients. The results showed MKLP1 was significantly up-regulated in TNBC tissues (Fig. 1H).

\section{MKLP1 promotes TNBC cells proliferation}


We performed KEGG pathway analysis on co-expressing genes with MKLP1, and found that these coexpressing genes were mainly enriched in the cell cycle pathway (Fig. S2A and Table S1). In addition, by analyzing a single cell database, we found that MKLP1 was associated with cell cycle and proliferation in a variety of tumors (Fig. S1B). In breast cancer datasets, MKLP1 expression was significantly related to cell cycle and proliferation, which was confirmed by several datasets (Fig. S2C). Moreover, the expression of MKLP1 in patients without lymph node metastasis was significantly lower than that in patients with lymph node metastasis (Fig. S2D). We also performed GSEA analysis after grouping TCGA patients according to the expression of MKLP1 and found MKLP1 might activate cell cycle pathway (Fig. S2E-F). Cells were transfected with lentiviral vectors containing shRNA-targeting MKLP1 (sh-MKLP1) or MKLP1 overexpression plasmids (MKLP1) respectively, with the efficiency confirmed by qRT-PCR and western blotting analysis (Fig. 2A-B). The growth curves derived from the CCK-8 assay showed that the cell proliferation rate was significantly reduced after the cells were transfected with sh-MKLP1 while cell proliferation was increased in response to MKLP1 upregulation (Fig. 2C-D). Similarly, colony formation assay indicated clonality of the TNBC cells was suppressed by MKLP1 downregulation but was markedly increased by MKLP1 overexpression (Fig. 2E-F). The EDU assay results indicated downregulated MKLP1 inhibited cell DNA replication ability while overexpression of MKLP1 caused reverse effects (Fig. 2G-H).

\section{MKLP1 facilitates TNBC cell invasion and migration.}

In the wound healing assay, the migration rate was dramatically inhibited in MKLP1 knockdown group but overexpression MKLP1 enforced TNBC cell migration rate (Fig. 3A-D). In the transwell assay, the number of migration and invasion cells was decreased after the downregulation of MKLP1 expression (Fig. 3E-F), while overexpression MKLP1 caused the reversed effect (Fig. 3G-H).

\section{MKLP1 promotes the epithelial-mesenchymal transition and $W n t / \beta$-catenin signaling pathway in TNBC}

Previous studies showed EMT progression plays a crucial role in TNBC [16, 38, 39]. We detected EMT marker proteins by western blotting. The results showed knockdown of MKLP1 decreased N-cadherin and vimentin levels and increased E-cadherin level (Fig. 4A), while overexpression of MKLP1 caused the opposite effects (Fig. 4B). Meanwhile, downregulated MKLP1 decreased Wnt/ $\beta$-catenin signaling pathway related genes expression, while upregulated MKLP1 reversed this effect (Fig. 4C-D).

\section{MKLP1 expression is directly regulated by FOXM1}

As transcription factors (TFs) play crucial roles in cancers [40]. We predicted TFs that might regulate the MKLP1 expression by bioinformatics methods. According to the prediction results, we analyzed the correlation between top 5 TFs and MKLP1 expression in TNBC (Fig. 5A and S3A-B). The result showed the expression of Foxm 1 and MKLP1 were the most relevant. Then we analyzed the motif of FOXM1 binding and whether it existed in the promoter region of MKLP1, the result demonstrated there was a binding peak of FOXM1 in the promoter region of MKLP1 (Fig. S3C-D). The CHIP analysis also marked 
the FOXM1 occupancy at MKLP1 promoter (Fig. 5B). In addition, the MKLP1 expression was detected in FOXM1 knockdown or overexpression TNBC cell lines by qRT-PCR and Western blotting. MKLP1 expression was decreased after knockdown of FOXM1, while FOXM1 overexpression reversed the effect (Fig. 5C-F). In conclusion, these results illustrated FOXM1 could bind to MKLP1 promoter region to promote MKLP1 expression.

\section{FOXM1 enhance TNBC cells proliferation, migration and invasion by regulating MKLP1 expression.}

Previous studies showed FOXM1 plays a crucial role in TNBC and acts as a specific marker for TNBC. Downregulated FOXM1 by tansfecting siRNA or using thiostrepton inhibited TNBC cells proliferation, migration and invasion (Fig. S4). We next explored whether MKLP1 was involved in the function of FOXM1 in TNBC cells. We found overexpression of MKLP1 could partly reverse the decrease in breast cancer cells proliferation caused by the knockdown of FOXM1 (Fig. 6A-E). Moreover, overexpression of MKLP1 could promote TNBC cells migration and invasion abilities inhibited by the downregulated FOXM1 (Fig. 6F-K). These results suggested MKLP1 was the target of FOXM1in promoting TNBC progression.

\section{WDR5 upregulates FOXM1 expression by accelerating H3K4me3 modification}

Histone modification plays an important role in the regulation of gene expression [41]. We explored whether histone modification affected FOXM1 expression. Through bioinformatics analysis, we found H3K4me3, which was reported to promote genes expression [42], might affect the FOXM1 expression (Fig. S5A). We then analyzed the correlation between components of methylation complex and FOXM1 expression in TNBC. The result showed the expression of Foxm1 and WDR5 were the most relevant (Fig. S5B-F). Public CHIP data showed a binding peak of H3K4me3 in the promoter region of FOXM1 and the CHIP analysis illustrated marked WDR5 and H3K4me3 occupancy at FOXM1 promoter (Fig. S5G-I). Moreover, knockdown of WDR5 inhibited FOXM1 and H3K4me3 expression (Fig. 7A-B). Downregulated WDR5 by transfecting siRNA or using OICR-9429, a small-molecule antagonist of the WDR5-MLL interaction, inhibited TNBC cells proliferation, migration and invasion (Fig. S6). We next explored whether FOXM1 was involved in the function of WDR5 in TNBC cells. The results demonstrated overexpression of FOXM1 partly reversed the decrease in breast cancer cells proliferation caused by knockdown of WDR5 (Fig. 7C-D). Meanwhile, overexpression rescued the inhibition of TNBC migration and invasion abilities caused by downregulated WDR5 (Fig. 7E-H). These results illustrated WDR5 promoted TNBC progression via upregulating FOXM1 expression.

\section{MKLP1 promotes TNBC proliferation and metastasis in vivo.}


To further investigate the role of MKLP1 in vivo, stable cell lines were injected subcutaneously into BALB/cA-nu mice. As shown in Fig. 8A-F, tumor sizes and weights of MKLP1 knockdown group were significantly lower than those of the control group. Moreover, we analyzed Ki-67 expression by IHC and lung metastasis by H\&E. The results indicated knockdown of MKLP1 alleviated tumor growth and metastasis in vivo (Fig. 8G-H).

\section{FOXM1 regulates the EMT progression and Wnt/ $\beta$-catenin pathway via MKLP1.}

As knockdown of MKLP1 inhibited EMT progression and $\beta$-catenin pathway, we explored whether these effects were regulated by FOXM1. Moreover, downregulated FOXM1 decreased the expression of EMT and Wnt/ $\beta$-catenin pathway related genes, but overexpression of MKLP1 could reverse the effect (Fig. 8IJ). Altogether, FOXM1, upregulated by WDR5 via H3K4me3 modification, regulated the EMT progression and Wnt/ $\beta$-catenin pathway by regulating MKLP1 expression (Fig. 9).

\section{Discussion:}

TNBC is a subtype of breast cancer with high heterogeneity, high recurrence, early metastasis and poor prognosis [4]. Due to the lack of hormone receptors and HER-2, endocrine therapy, targeted therapy and chemotherapy often fail to be effective treatments for TNBC $[43,44]$. It is urgent to find potential molecular targets to improve the prognosis of TNBC. In our study, we found MKLP1 was upregulated in TNBC and associated with prognosis of TNBC by bioinformatic analysis. Several studies showed MKLP1 plays an oncogenic role in several cancer types $[11,45]$. Moreover, our results illustrated MKLP1 was upregulated in TNBC cell lines and tissues. Knockdown of MKLP1 inhibited TNBC proliferation and metastasis while upregulated MKLP1 caused opposite effects. Previous studies have demonstrated Wnt/ $\beta$-catenin signaling pathway is involved in different types of tumors progression [20,46], including breast cancer [47, 48], gastric cancer [11], hepatocellular carcinoma [49], lung cancer [50]. And aberrant Wnt signaling pathway was associated with tumor proliferation, metastasis, stemness and drug resistance $[23,24,51,52]$. It was reported MKLP1 could activate Wnt/ $\beta$-catenin signaling pathway in gastric and colorectal cancer $[11,45]$, and then we explored whether MKLP1 affected Wnt/ $\beta$-catenin signaling pathway in TNBC. Our results showed the related genes of Wnt/ $\beta$-catenin signaling pathway, such as $\beta$ catenin, CyclinD1 and c-myc, were upregulated due to the MKLP1 overexpression, indicating MKLP1 promotes TNBC progression via activating this pathway. In addition, Wnt/ $\beta$-catenin signaling pathway can promote EMT progression [49], which is associated with tumor progression [23,50]. We also found knockdown of MKLP1 decreased EMT marker proteins expression, suggesting MKLP1 could promote EMT progression in TNBC.

FOXM1 is a transcription factor and plays a crucial role in different types of cancers, including gastric cancer, lung cancer and prostate cancer [53-56]. In breast cancer, FOXM1 was identified as a specific marker for TNBC and enhanced paclitaxel resistance in TNBC $[57,58]$. In the present study, we found 
FOXM1 might be recruited and bind to the promoter region of MKLP1 via analyzing public CHIP-Seq datasets and FOXM1 expression is positively correlated with MKLP1 expression in TNBC tissues. Inhibition of FOXM1 with siRNA or thiostrepton [59] significantly suppressed TNBC cells proliferation and migration, while overexpression of MKLP1 could partly rescue the effects caused by FOXM1 inhibition. Meanwhile, FOXM1 could promote EMT progression and activate Wnt/ $\beta$-catenin pathway, which is in accord with previous study [60]. Thus, FOXM1 could accelerate TNBC progression via regulating transcriptional activity of MKLP1.Additionaly, thiostrepton, the selective inhibitor of FOXM1, might be the potential therapy drug for TNBC.

Epigenetics refers to the reversible and heritable changes in gene expression and function without changes in the DNA sequence, including DNA modification, various modifications of histones, and so on [61]. H3K4me3 modification is a common modification of histones, which affects transcription of genes[41]. In the present study, we detected histone modification of FOXM1 by bioinformatics analysis and found H3K4me3 modification might affect FOXM1 expression. Subsequent experiments demonstrated WDR5 upregulated FOXM1 expression via promoting FOXM1 H3K4me3 modification. In addition, the effects caused by inhibition of WDR5 on proliferation and metastasis of TNBC cells could be partly restored by the upregulating FOXM1 expression. Our results illustrated WDR5 increased H3K4me3 modification to upregulate FOXM1 expression. Knockdown of WDR5 or small molecule antagonist of WDR5-MLL complex could significantly inhibit TNBC cells proliferation and metastasis, suggesting OICR9429 might be used to treat TNBC.

\section{Conclusion}

In conclusion, our results demonstrated MKLP1 plays a crucial role in TNBC progression. Mechanistically, MKLP1 promotes TNBC proliferation and metastasis abilities via activating Wnt/ $\beta$-catenin pathway and promoting EMT progression. Meanwhile, FOXM1, upregulated by WDR5 via H3K4me3 modification, promotes MKLP1 expression through enhancing transcription of MKLP1. Our findings elucidate WDR5/FOXM1/MKLP1/Wnt/ $\beta$-catenin axis is associated with TNBC progression and may provide a novel and promising therapeutic target. Accordingly, thiostrepton and OICR-9429 alone or in combination have the potential to treat TNBC.

\section{Abbreviations}

TNBC: Triple negative breast cancer; MKLP1: Mitotic kinesin-like protein 1; TCGA: The Cancer Genome Atlas; FOXM1: Forkhead Box M1; WDR5: WD Repeat Domain 5; EMT: Epithelial-mesenchymal transition; qRT-PCR: RNA isolation and quantitative real time-polymerase chain reaction; CCK8: Cell Counting Kit-8; EdU: 2.8 5-ethynyl-2'-deoxyuridine; CHIP: Chromatin immunoprecipitation assay

\section{Declarations}

\section{Ethics approval and consent to participate}


All animal experiments were approved by the Animal Management Committee of Nanjing Medical University. All procedures involving human participants in this study were approved by the Research Ethics Committee of The First Affiliated Hospital of Nanjing Medical University. All patients included in the present study signed informed consent

\section{Consent for publication}

All authors provided their consent to publish the study.

\section{Availability of data and materials}

The datasets generated during and/or analysed during the current study are available in Gene Expression Omnibus (GEO) database.

\section{Competing interests}

The authors declare no competing interest.

\section{Funding}

This work was supported by the National Natural Science Foundation of China $(81972486,81802748$, 81802644), the Key Medical Talents of Jiangsu Province (ZDRCA2016029) and '333' High-level Talents Training Project of Jiangsu Province (BRA2016505). Besides, the work was also funded by the International Cooperation Project of Jiangsu Provincial Science and Technology Department (BZ2018054) and the Priority Academic Program Development of Jiangsu Higher Education Institutions (PAPD)(JX10231801)

\section{Authors' contributions}

ZL, JF W, GR Z and QD designed the experiments. ZL, HY Y and XL Z carried out the experiments and acquired the data. ZL, LS and $X Z$ analyzed and interpreted the data. YZ H and HY Y analyzed the TCGA and GEO dataset. HY Y and XY D were responsible for clinical sample collection. JF W and QD conceived of the study, designed experiments, helped interpret data, and revised the draft manuscript. All authors reviewed and approved the final manuscript

\section{Acknowledgments}

Not applicable

\section{References}

1. DeSantis CE, Ma J, Gaudet MM, Newman LA, Miller KD, Goding Sauer A, et al. Breast cancer statistics, 2019. CA Cancer J Clin. 2019; 69: 438-451.

2. Siegel RL, Miller KD, Fuchs HE, Jemal A. Cancer Statistics, 2021. CA Cancer J Clin. 2021; 71: 7-33. 
3. Eroles P, Bosch A, Pérez-Fidalgo JA, Lluch A. Molecular biology in breast cancer: intrinsic subtypes and signaling pathways. Cancer Treat Rev. 2012; 38: 698-707.

4. Dent R, Trudeau M, Pritchard KI, Hanna WM, Kahn HK, Sawka CA, et al. Triple-negative breast cancer: clinical features and patterns of recurrence. Clin Cancer Res. 2007; 13: 4429-4434.

5. Liu X, Gong H, Huang K. Oncogenic role of kinesin proteins and targeting kinesin therapy. Cancer Sci. 2013; 104: 651-656.

6. Goldstein LS, Philp AV. The road less traveled: emerging principles of kinesin motor utilization. Annu Rev Cell Dev Biol. 1999; 15: 141-183.

7. Pavicic-Kaltenbrunner V, Mishima M, Glotzer M. Cooperative assembly of CYK-4/MgcRacGAP and ZEN-4/MKLP1 to form the centralspindlin complex. Mol Biol Cell. 2007; 18: 4992-5003.

8. van de Ven RA, de Groot JS, Park D, van Domselaar R, de Jong D, Szuhai K, et al. p120-catenin prevents multinucleation through control of MKLP1-dependent RhoA activity during cytokinesis. Nat Commun. 2016; 7: 13874.

9. Fischer M, Grundke I, Sohr S, Quaas M, Hoffmann S, Knörck A, et al. p53 and cell cycle dependent transcription of kinesin family member 23 (KIF23) is controlled via a CHR promoter element bound by DREAM and MMB complexes. PLoS One. 2013; 8: e63187.

10. Liang W, Liu X, Huang H, Gao Z, Li K. Prognostic significance of KIF23 expression in gastric cancer. World J Gastrointest Oncol. 2020; 12: 1104-1118.

11. Liu Y, Chen H, Dong P, Xie G, Zhou Y, Ma Y, et al. KIF23 activated Wnt/ $\beta$-catenin signaling pathway through direct interaction with Amer1 in gastric cancer. Aging (Albany NY). 2020; 12: 8372-8396.

12. Kato T, Wada H, Patel P, Hu H, Lee D, Ujiie H, et al. Overexpression of KIF23 predicts clinical outcome in primary lung cancer patients. Lung Cancer. 2016; 92: 53-61.

13. Gao C, Ren J, Yu J, Li S, Guo X, Zhou Y. KIF23 enhances cell proliferation in pancreatic ductal adenocarcinoma and is a potent therapeutic target. Ann Transl Med. 2020; 8: 1394.

14. Yao D, Song Q, He X. Kinesin family member 23 (KIF23) contributes to the progression of bladder cancer cells in vitro and in vivo. Neoplasma. 2020; 68: 298-306.

15. Brabletz T, Kalluri R, Nieto MA, Weinberg RA. EMT in cancer. Nat Rev Cancer. 2018; 18: 128-134.

16. Zeng $D$, Liang $Y$, Xiao $Y$, Wei $X$, Lin H, Wu Y, et al. Inhibition of Notch1 reverses EMT and chemoresistance to cisplatin via direct downregulation of MCAM in triple-negative breast cancer cells. Int J Cancer. 2020; 147: 490-504.

17. Kim HY, Kim YM, Hong S. DNAJB9 suppresses the metastasis of triple-negative breast cancer by promoting FBXO45-mediated degradation of ZEB1. Cell Death Dis. 2021; 12: 461.

18. Fresno Vara JA, Casado E, de Castro J, Cejas P, Belda-Iniesta C, González-Barón M. PI3K/Akt signalling pathway and cancer. Cancer Treat Rev. 2004; 30: 193-204.

19. O'Shea JJ, Schwartz DM, Villarino AV, Gadina M, Mclnnes IB, Laurence A. The JAK-STAT pathway: impact on human disease and therapeutic intervention. Annu Rev Med. 2015; 66: 311-328. 
20. Nusse R, Clevers H. Wnt/ $\beta$-Catenin Signaling, Disease, and Emerging Therapeutic Modalities. Cell, 2017; 169: 985-999.

21. Briscoe J, Thérond PP. The mechanisms of Hedgehog signalling and its roles in development and disease. Nat Rev Mol Cell Biol. 2013; 14: 416-429.

22. Bugter JM, Fenderico N, Maurice MM. Mutations and mechanisms of WNT pathway tumour suppressors in cancer. Nat Rev Cancer. 2021; 21: 5-21.

23. Zhou P, Li Y, Li B, Zhang M, Liu Y, Yao Y, et al. NMIIA promotes tumor growth and metastasis by activating the Wnt/ $\beta$-catenin signaling pathway and EMT in pancreatic cancer. Oncogene. 2019; 38: 5500-5515.

24. Peng K, Kou L, Yu L, Bai C, Li M, Mo P, et al. Histone Demethylase JMJD2D Interacts With $\beta$-Catenin to Induce Transcription and Activate Colorectal Cancer Cell Proliferation and Tumor Growth in Mice. Gastroenterology. 2019; 156: 1112-1126.

25. Li Y, Wang Z, Su P, Liang Y, Li Z, Zhang H, et al. Circ-EIF6 encodes EIF6-224aa to promote TNBC progression via stabilizing MYH9 and activating Wnt/beta-catenin pathway. Mol Ther. 2021.

26. Kong Y, Ou X, Li X, Zeng Y, Gao G, Lyu N, et al. LGR6 Promotes Tumor Proliferation and Metastasis through Wnt/beta-Catenin Signaling in Triple-Negative Breast Cancer. Mol Ther Oncolytics. 2020; 18 : 351-359.

27. Yu L, Wang C, Pan F, Liu Y, Ren X, Zeng H, et al. HePTP promotes migration and invasion in triplenegative breast cancer cells via activation of Wnt/beta-catenin signaling. Biomed Pharmacother. 2019; 118: 109361.

28. King TD, Suto MJ, Li Y. The Wnt/beta-catenin signaling pathway: a potential therapeutic target in the treatment of triple negative breast cancer. J Cell Biochem. 2012; 113: 13-18.

29. Chandrashekar DS, Bashel B, Balasubramanya SAH, Creighton CJ, Ponce-Rodriguez I, Chakravarthi B, et al. UALCAN: A Portal for Facilitating Tumor Subgroup Gene Expression and Survival Analyses. Neoplasia. 2017; 19: 649-658.

30. Yuan H, Yan M, Zhang G, Liu W, Deng C, Liao G, et al. CancerSEA: a cancer single-cell state atlas. Nucleic Acids Res. 2019; 47: D900-d908.

31. Liu J, Dong C, Liu Y, Wu H. CGPE: An integrated online server for Cancer Gene and Pathway Exploration. Bioinformatics. 2020:37: 2201-2202.

32. Li T, Fu J, Zeng Z, Cohen D, Li J, Chen Q, et al. TIMER2.0 for analysis of tumor-infiltrating immune cells. Nucleic Acids Res. 2020; 48: W509-W514.

33. Liu T, Ortiz JA, Taing L, Meyer CA, Lee B, Zhang Y, et al. Cistrome: an integrative platform for transcriptional regulation studies. Genome Biol. 2011; 12: R83.

34. Zeng J, Li G. TFmapper: A Tool for Searching Putative Factors Regulating Gene Expression Using ChIP-seq Data. Int J Biol Sci. 2018; 14: 1724-1731.

35. Xi P, Zhang X, Zhu L, Dai X, Cheng L, Hu Y, et al. Oncogenic action of the exosome cofactor RBM7 by stabilization of CDK1 mRNA in breast cancer. NPJ Breast Cancer. 2020; 6: 58. 
36. Zhu L, Xi P, Li X, Sun X, Zhou W, Xia T, et al. The RNA binding protein RBMS3 inhibits the metastasis of breast cancer by regulating Twist1 expression. J Exp Clin Cancer Res, 2019; 38: 105.

37. Wu J, Zhou X, Sun X, Xia T, Li X, Shi L, et al. RBM38 is involved in TGF- $\beta$-induced epithelial-tomesenchymal transition by stabilising zonula occludens- 1 mRNA in breast cancer. $\mathrm{Br} \mathrm{J}$ Cancer. 2017; 117: 675-684.

38. Miao K, Lei JH, Valecha MV, Zhang A, Xu J, Wang L, et al. NOTCH1 activation compensates BRCA1 deficiency and promotes triple-negative breast cancer formation. Nat Commun. 2020; 11: 3256.

39. Vijay GV, Zhao N, Den Hollander P, Toneff MJ, Joseph R, Pietila M, et al. GSK3 $\beta$ regulates epithelialmesenchymal transition and cancer stem cell properties in triple-negative breast cancer. Breast Cancer Res. 2019; 21: 37.

40. Lambert SA, Jolma A, Campitelli LF, Das PK, Yin Y, Albu M, et al. The Human Transcription Factors. Cell. 2018; 172: 650-665.

41. Audia JE, Campbell RM. Histone Modifications and Cancer. Cold Spring Harb Perspect Biol. 2016; 8: a019521.

42. Lu C, Liu Z, Klement JD, Yang D, Merting AD, Poschel D, et al. WDR5-H3K4me3 epigenetic axis regulates OPN expression to compensate PD-L1 function to promote pancreatic cancer immune escape. J Immunother Cancer. 2021; 9: e002624.

43. Bianchini G, Balko JM, Mayer IA, Sanders ME, Gianni L. Triple-negative breast cancer: challenges and opportunities of a heterogeneous disease. Nat Rev Clin Oncol. 2016; 13: 674-690.

44. Garrido-Castro AC, Lin NU, Polyak K. Insights into Molecular Classifications of Triple-Negative Breast Cancer: Improving Patient Selection for Treatment. Cancer Discov. 2019; 9: 176-198.

45. Ji Z, Mi A, Li M, Li Q, Qin C. Aberrant KIF23 expression is associated with adverse clinical outcome and promotes cellular malignant behavior through the Wnt/ $\beta$-catenin signaling pathway in Colorectal Cancer. J Cancer. 2021; 12: 2030-2040.

46. Xu X, Zhang M, Xu F, Jiang S. Wnt signaling in breast cancer: biological mechanisms, challenges and opportunities. Mol Cancer. 2020; 19: 165.

47. Ren L, Chen H, Song J, Chen X, Lin C, Zhang X, et al. MiR-454-3p-Mediated Wnt/beta-catenin Signaling Antagonists Suppression Promotes Breast Cancer Metastasis. Theranostics. 2019; 9(2): 449-465.

48. Cai J, Guan H, Fang L, Yang Y, Zhu X, Yuan J, et al. MicroRNA-374a activates Wnt/beta-catenin signaling to promote breast cancer metastasis. J Clin Invest. 2013; 123: 566-579.

49. Zhao Y, Wang J, Xu C, Li Y, Sun B, Yang L. HEG1 indicates poor prognosis and promotes hepatocellular carcinoma invasion, metastasis, and EMT by activating Wnt/ $\beta$-catenin signaling. Clin Sci (Lond). 2019; 133: 1645-1662.

50. Li H, Ke F, Lin C, Lu M, Kuo Y, Wang Y, et al. ENO1 Promotes Lung Cancer Metastasis via HGFR and WNT Signaling-Driven Epithelial-to-Mesenchymal Transition. Cancer Res. 2021; 81: 4094-4109. 
51. Lu Y, Zhao X, Liu Q, Li C, Graves-Deal R, Cao Z, et al. IncRNA MIR100HG-derived miR-100 and miR125b mediate cetuximab resistance via Wnt/ $\beta$-catenin signaling. Nat Med. 2017; 23: 1331-1341.

52. Esposito M, Mondal N, Greco TM, Wei Y, Spadazzi C, Lin SC, et al. Bone vascular niche E-selectin induces mesenchymal-epithelial transition and Wnt activation in cancer cells to promote bone metastasis. Nat Cell Biol. 2019; 21: 627-639.

53. Xu M, Wang Y, Weng W, Wei P, Qi P, Zhang Q, et al. A Positive Feedback Loop of IncRNA-PVT1 and FOXM1 Facilitates Gastric Cancer Growth and Invasion. Clin Cancer Res. 2017; 23: 2071-2080.

54. Iltzsche F, Simon K, Stopp S, Pattschull G, Francke S, Wolter P, et al. An important role for Myb-MuvB and its target gene KIF23 in a mouse model of lung adenocarcinoma. Oncogene. 2017; 36: 110-121.

55. Bella L, Zona S, Nestal de Moraes G, Lam EW. FOXM1: A key oncofoetal transcription factor in health and disease. Semin Cancer Biol. 2014; 29: 32-39.

56. Lin J, Wang W, Hu T, Zhu G, Li L, Zhang C, et al. FOXM1 contributes to docetaxel resistance in castration-resistant prostate cancer by inducing AMPK/mTOR-mediated autophagy. Cancer Letters. 2020; 469: 481-489.

57. Tan Y, Wang Q, Xie Y, Qiao X, Zhang S, Wang Y, et al. Identification of FOXM1 as a specific marker for triple-negative breast cancer. International Journal of Oncology. 2019; 54: 87-97.

58. Yang N, Wang C, Wang J, Wang Z, Huang D, Yan M, et al. Aurora kinase A stabilizes FOXM1 to enhance paclitaxel resistance in triple-negative breast cancer. J Cell Mol Med. 2019; 23: 6442-6453.

59. Kwok JM, Myatt SS, Marson CM, Coombes RC, Constantinidou D, Lam EW. Thiostrepton selectively targets breast cancer cells through inhibition of forkhead box M1 expression. Mol Cancer Ther. 2008; 7: 2022-2032.

60. Gong A, Huang S. FoxM1 and Wnt/beta-catenin signaling in glioma stem cells. Cancer Res. 2012; 72 : 5658-5662.

61. Dawson MA, Kouzarides T. Cancer epigenetics: from mechanism to therapy. Cell. 2012; 150: 12-27.

\section{Figures}

\section{Figure 1}

MKLP1 is overexpressed in TNBC and associated with poor prognosis. A. The Venn-diagram showed the upregulated genes in TNBC. B. Expression of MKLP1 in 52 breast cancer cell lines from public microarray dataset (GSE41313). C. Expression of MKLP1 in luminal breast cancer and TNBC from public microarray dataset (GSE5460). D-E: MKLP1 mRNA (D) and protein (E) expression in different subtypes of breast cancer based on TCGA and CPTAC database. F. Kaplan-Meier survival analysis showed the correlation between the expression of MKLP1 and the relapse free survival of TNBC patients based on TCGA. G. Expression of MKLP1 in the breast cancer cell lines and MCF-10A. H. Expression of MKLP1 in TNBC 
tissues and adjacent normal tissues. The relative quantification was calculated by the $2^{-\Delta \Delta \mathrm{Ct}}$ method and normalized based on GAPDH. Data were shown as mean \pm SD. ${ }^{*} p<0.05,{ }^{*} p<0.01, * \star \star p<0.001$.

\section{Figure 2}

MKLP1 promotes the proliferation of TNBC cells. A-B: qRT-PCR and western blot were used to verify the efficiency of MKLP1 knockdown (A) and overexpression (B) in MDA-MB-231 and SUM1315 cells. C-D: Cell proliferation was determined by CCK-8 assays in MDA-MB-231 and SUM1315 cells after knockdown (C) or overexpression (D) of MKLP1. E-F: The colony formation results of MDA-MB-231 and SUM1315 cells after knockdown (E) or overexpression (F) of MKLP1. Colonies $>50 \mathrm{~mm}$ were counted. G-H: EdU assays results of MDA-MB-231 and SUM1315 cells after knockdown (G) or overexpression (H) of MKLP1. Blue indicates DAPI, red indicates EdU. Data were shown as mean \pm SD. ${ }^{\star} p<0.05,{ }^{\star \star} p<0.01,{ }^{\star \star *} p<0.001$.

\section{Figure 3}

MKLP1 enhances the migration and invasion of TNBC cells. A-D: The wound healing assays were performed to assess the effect of knockdown (A-B) or overexpression (C-D) of MKLP1 on cell motility at 0 and 24h in MDA-MB-231 and SUM1315 cells. E-H: The representative images of migrated and invaded MDA-MB-231 and SUM1315 cells after MKLP1 knockdown (E-F) or overexpression (G-H). Data were shown as mean $\pm S D, * p<0.05, * * p<0.01, * \star * p<0.001$.

\section{Figure 4}

MKLP1 enhances EMT progression and activates Wnt/ $\beta$-catenin signaling pathway. A-B: Western blot was used to detect protein level of biomarkers of EMT in MDA-MB-231 and SUM1315 cells after knockdown (A) or overexpression (B) of MKLP1. C-D: Western blot was used to detect protein level of biomarkers of Wnt/ $\beta$-catenin signaling pathway in MDA-MB-231 and SUM1315 cells after knockdown (C) or overexpression (D) of MKLP1.

\section{Figure 5}

FOXM1 directly binds to MKLP1 promoter to regulate MKLP1 expression. A. The correlation between expression of FOXM1 and MKLP1. B. CHIP assays were performed in MDA-MB-231 and SUM1315 cells. C-F: qRT-PCR and western blot were used to verify the effect of FOXM1 knockdown (C-D) or 
overexpression (E-F) on MKLP1 expression in MDA-MB-231 and SUM1315 cells. Data were shown as mean $\pm S D,{ }^{\star} p<0.05,{ }^{\star *} p<0.01, \star \star \star p<0.001$.

\section{Figure 6}

MKLP1 partly restores the effect caused by FOXM1 in TNBC cells. A-E: CCK-8 assays (A), colony formation assays (B-C) and EdU assays (D-E) indicated MKLP1 could partly rescue the repressive effect of knockdown of FOXM1 on cell proliferation. F-K: The wound healing assays (F-G) and transwell assays $(\mathrm{H}-\mathrm{K})$ demonstrated the overexpression of MKLP1 partly reversed the repressive effect on cell migration and invasion caused by knockdown of FOXM1. Data were shown as mean $\pm S D$, ${ }^{\star} p<0.05,{ }^{\star \star} p<0.01$, $\star \star \star p<0.001$.

\section{Figure 7}

WDR5 regulates TNBC cells biological function via regulating FOXM1. A. qRT-PCR was used to verify the expression of WDR5, FOXM1 and MKLP1 after transfected with si-WDR5. B. Western blotting was used to verify the expression of WDR5, FOXM1, MKLP1 and H3K4me3 after transfected with si-WDR5. C-D: CCK-8 assays (C) and colony formation assays indicated FOXM1 could partly rescue the repressive effect of knockdown of WDR5 on cell proliferation. E-H: The transwell assays demonstrated the overexpression of FOXM1 partly reversed the repressive effect on cell migration and invasion caused by knockdown of WDR5 in MDA-MB-231 (E-F) and SUM1315 (G-H). Data were shown as mean \pm SD, ${ }^{*} p<0.05,{ }^{\star *} p<0.01$, $\star \star \star p<0.001$.

\section{Figure 8}

Knockdown of MKLP1 inhibits TNBC progression in vivo by targeting Wnt/ $\beta$-catenin signaling pathway.

A-F: Representative images of xenograft tumors, tumor sizes and weights of MKLP1 knockdown (shMKLP1) and control (sh-NC) group. G-H: Ki-67 expression was analyzed by IHC and lung metastasis nodes were analyzed by H\&E. I-J: Western blotting was used to determine the protein expression of genes that related to the pathways after transfecting with si-FOXM1 and protein expression level of these genes was detected after the restoration of MKLP1. Data were shown as mean $\pm S D,{ }^{*} p<0.05, * \star p<0.01$, $\star \star \star p<0.001$.

\section{Figure 9}


Molecular mechanism of WDR5/FOXM1/MKLP1/Wnt/ $\beta$-catenin axis in the progression in triple negative breast cancer.

\section{Supplementary Files}

This is a list of supplementary files associated with this preprint. Click to download.

- FigureS1.tif

- Figures2.tif

- FigureS3.tif

- FigureS4.tif

- FigureS5.tif

- FigureS6.tif

- SupplementFigureLegends.docx

- SupplementTable.docx 